\title{
Performance Evaluation of Image Enhancement Techniques in Spatial and Wavelet Domains
}

\author{
Lovely Passrija \\ Deptt. of ECE \\ Faridkot,Punjab \\ India
}

\author{
Amardeep Singh Virk \\ Deptt. of ECE \\ Faridkot,Punjab \\ India
}

\author{
Mandeep Kaur \\ Deptt. of ECE \\ Faridkot,Punjab \\ India
}

\begin{abstract}
Denoising is one of the important tasks in image processing. Despite the significant research conducted on this topic, the development of efficient denoising methods is still a compelling challenge.

In this paper, spatial domain methods and Wavelet Domain Methods of image denoising have been evaluated. The medical ultrasound images suffer from speckle noise which is multiplicative in nature and more difficult to remove than additive noise. In the spatial filter methods Median Filter and Wiener Filter are implemented. These methods are based on the simple formulas that are proposed by different authors. In Wavelet Methods Visu Shrink, Neigh shrink and Bayes Shrink are implemented. The basic idea of wavelet methods is to denoise the image by applying wavelet transform to the noisy image, then thresholding the detailed wavelet coefficient and inverse transforming the set of thresholded coefficient to obtain the denoised image.

The comparison of all filters methods is done using various Quality Metrics like Peak Signal-to-Noise Ratio (PSNR), Bit Error Rate (BER), Mean Square Error, etc.

The filters methods implemented in MATLAB 7.10.0.499(R2010a).
\end{abstract}

Keywords: Additive White Gaussian Noise, Speckle Noise, Hybrid, Median, Wavelets

\section{INTRODUCTION 1.1 ULTRASOUND IMAGES}

Ultrasonography is considered to be one of the most powerful technique for imaging organs and soft tissue structures in human body.

It is preferred over other medical imaging methods because it is:

1. Non-invasive

2. Portable

3. Versatile

4. Low cost

Despite their obvious advantages, ultrasound(US) images are contaminated with multiplicative noise called 'speckle' which is one of the major sources of image quality degradation.

In the medical literature, speckle has been treated as a distracting artifact as it tends to degrade the resolution and the object detection ability. Moreover, in US images the speckle noise has a spatial correlation length on each axis, which is same as resolution cell size. This spatial correlation makes the speckle suppression a very difficult and delicate task, hence, a trade-off has to be made between the degree of speckle suppression and feature preservation [1].

\subsection{JPEG IMAGE}

Jpeg pronounced as jay-peg is a commonly used method of lossy compression for digital photography (image). The degree of compression can be adjusted, allowing a selectable trade off between storage size and image quality. JPEG typically achieves 10:1 compression with little perceptible loss in image quality.

JPEG compression is used in a number of image file formats. JPEG/Exif is the most common image format used by digital cameras and other photographic image capture devices; along with JPEG/JFIF, it is the most common format for storing and transmitting photographic images on the World Wide Web. These format variations are often not distinguished, and are simply called JPEG. The term "JPEG" is an acronym for the Joint Photographic Experts Group which created the standard. The MIME media type for JPEG is image/jpeg (defined in RFC 1341), except in Internet Explorer, which provides a MIME type of image/jpeg when uploading JPEG images [2].

It supports a maximum image size of $65535 \times 65535[3]$.

\subsection{SPECKLE NOISE}

Speckle significantly degrades the image quality and hence, makes it more difficult for the observer to discriminate fine detail of the images in diagnostic examinations [4]. Speckle is a form of multiplicative noise, which makes visual interpretation difficult [5]. Laser holography and ultrasound imaging are two techniques susceptive to speckle degradation. Speckle noise causing greater degradation within bright areas of an image than in dark areas.

\subsection{GAUSSIAN NOISE}

Gaussian noise is evenly distributed over the signal [4]. Noise found in digital images is additive with uniform power and Gaussian probability distribution. Such a noise is called Additive White Gaussian Noise(AWGN). This means that each pixel in the noisy image is the sum of the true pixel value and a random Gaussian distributed noise value. As the name indicates, this type of noise has a Gaussian distribution, which has a bell shaped probability distribution function given by,

$$
F(g)=\frac{1}{\sqrt{2 \pi \sigma^{2}}} e^{-(g-m)^{2} / 2 \sigma^{2}}
$$

where $g$ represents the gray level, $m$ is the mean or average of the function and $\sigma$ is the standard deviation of the noise. Graphically, it is represented as shown in Figure 1.4. When introduced into an image, Gaussian noise with zero mean and variance as 0.05 would look as in Image 1.4 (a). Image 1.4 (b) illustrates the Gaussian noise with mean (variance) as 1.5 (10) over a base image with a constant pixel value of 100 . 


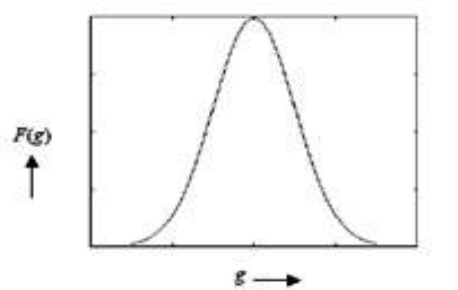

Figure1.4 Gaussian distribution

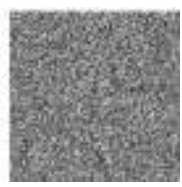

Figure 1.4(a)

Gaussian noise, $($ mean $=0$, variance 0.05 )

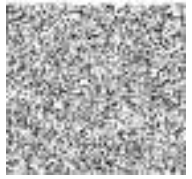

Figure 1.4 (b)

Gaussian noise, $($ mean $=1.5$, variance 10$)$

\section{IMAGE DENOISING}

De-noising plays a vital role in the field of the image preprocessing. It attempts to remove whatever noise is present and retains the significant information, regardless of the frequency contents of the signal. High frequency noise can easily be removed by linear and non linear filtering, it is challenging to remove low frequency noise because it is difficult to distinguish between real signal and low frequency noise .Ultrasonography is often preferred over other medical imaging modalities because it is noninvasive, portable, and versatile, it does not use ionizing radiations and it is relatively of low-cost. A common problem with the interpretation of Jpeg \& ultrasound images though, is the presence of speckle noise. The goal of denoising is to remove the noise while retaining as much as possible the important image features. Image denoising still remains the challenge for researchers because noise removal introduces artifacts and causes blurring of the images [5].

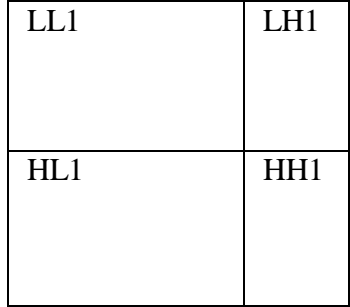

Figure 3.2 (a) One-Level

\begin{tabular}{|l|l|l|}
\hline LL2 & LH2 & \multirow{2}{*}{ LH1 } \\
HL2 & HH2 & \\
\hline HL1 & & HH1 \\
& & \\
\hline
\end{tabular}

(b) Two-Level
One of the widespread methods which is mainly exploited for speckle reduction is the wavelet shrinkage method. A comparative study between wavelet coefficient shrinkage filter and several standard speckle filters that are being largely used for speckle noise suppression which shows that the wavelet-based approach is deployed among the best for speckle removal [1].

\section{WAVELETS}

Different techniques are available for image denoising as wavelets which are multi resolution technique which provides better result for the original image. The motivation is that as the wavelet transform is good at energy compaction, the small coefficients are more likely due to noise and large coefficient due to important signal features. These small coefficients can be threshold without affecting the significant features of the image. Low frequency noise components are dealt with spatial domain filtering while the wavelet thresholding technique is designed to deal with high frequency noise components.
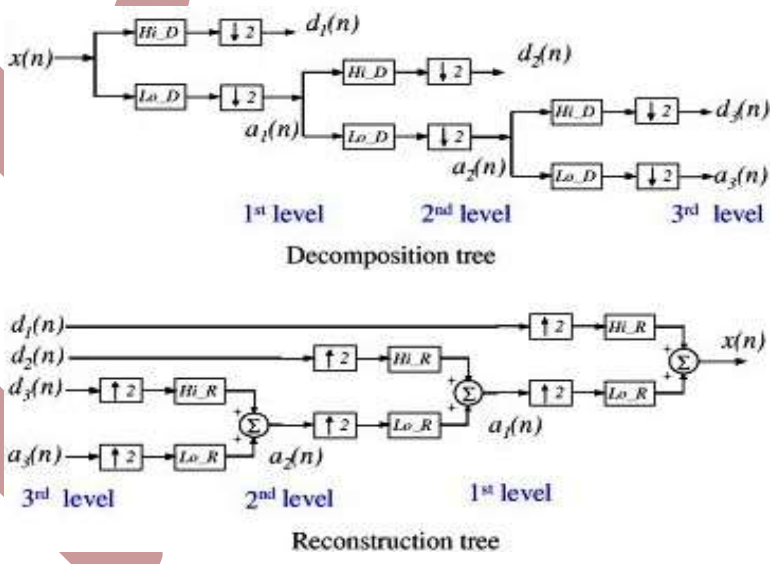

Figure 3.1 3-level Wavelet Decomposition and Reconstruction tree.

\section{DECOMPOSITION:}

Decomposition provides sub bands corresponding to different resolution levels and orientation. These sub bands labeled LH1, HL1 and HH1 represent the finest scale wavelet coefficients i.e., detail images while the sub band LL1 corresponds to coarse level coefficients i.e., approximation image.

To obtain the next coarse level of wavelet coefficients, the sub band LL1 alone is further decomposed and critically sampled using similar filter bank shown in fig 3.2 (a). This results in twolevel wavelet decomposition is as shown in fig 3.2 (b).

\section{WAVELET FILTERS}

\section{VisuShrink}

VisuShrink was introduced by Donoho [6]. It uses a threshold value $t$ that is proportional to the standard deviation of the noise. It follows the hard thresholding rule. It is also referred to as universal threshold and is defined as 


$$
t=\sigma^{2} \sqrt{2 \log (n)}
$$

$\sigma^{2}$ is the noise variance present in the signal and $n$ represents the signal size or number of samples. An estimate of the noise level $\sigma$ was defined based on the median absolute deviation [18] given by

$$
\hat{\sigma}=\frac{\operatorname{median}\left(\left(\left\{\left|g_{j-1, k}\right|: k=0,1, \ldots 2^{j-1}-1\right\}\right)\right.}{0.6745}
$$

where $g_{j-1, k}$ corresponds to the detail coefficients in the wavelet transform.

\section{BayesShrink}

The goal of this method is to minimize the Bayesian risk, and hence its name, BayesShrink. It uses soft

thresholding and is subband-dependent, which means that thresholding is done at each band of resolution in the wavelet decomposition [7]. Like the SureShrink procedure, it is smoothness adaptive. The Bayes threshold, $t_{B}$ is defined as

$$
t_{B}=\sigma^{2} / \sigma_{s}^{2}
$$

where $\sigma^{2}$ is the noise variance and $\sigma_{s}{ }^{2}$ is the signal variance without noise.

\section{Experimental Results}

A. Images corrupted by additive white Gaussian noise (Sigma=15):

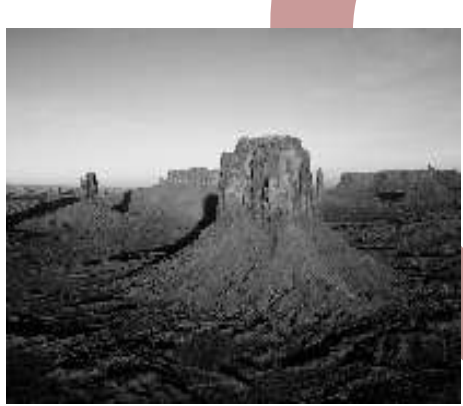

(a)

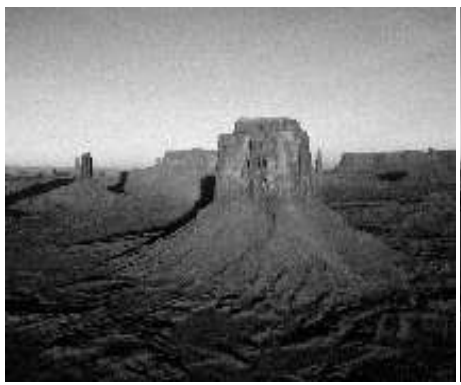

(d)

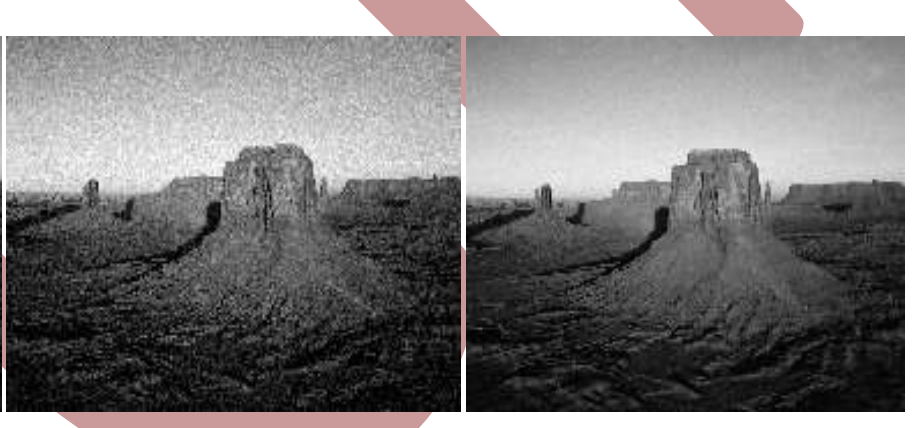

(b)

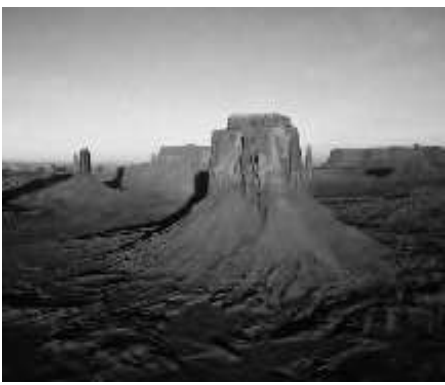

(e)

\section{HYBRID}

In this technique we are using two combinations of filters median filter and bayeshrink. We have seen PSNR value is increasing, the image quality is better than other. Since in hybrid technique performs the wavelet operations for denoising the image contents, time increases. Performance parameters like bit error rate, mean square error and time are evaluated. In this paper we explored this technique for additive white gaussian noise and speckle noise.

\section{RELATED RESEARCH WORK}

Various techniques are experimented for speckle noise reduction like lee, kuan, frost, SRAD, wiener and median filtering techniques in which speckle noise has been reduced by converting the multiplicative noise into additive noise for easy removal of the speckle noise from the image.

\section{Present Work}

We proposed a new model for denoising the image corrupted by additive white Gaussian noise and speckle noise. Speckle noise is multiplicative noise, it is difficult to get better results after denoising image. So we have used combinational techniques in wavelet and spatial domains to optimize the performance. Comparison of filters is carried out using different performance parameters like peak signal to noise ratio, bit error rate, mean square error and processing time. (a) Original Image (b) Noisy Image, method
Reconstructed images after applying (c) Bayeshrink

(d) Neighshrink (e) Hybrid 
Table showing the performance of different filters

\begin{tabular}{|l|l|l|l|l|}
\hline Wavelets & PSNR & BER & MSE & TIME \\
\hline Median filter & 33.1021 & & & \\
\hline Wiener filter & 32.8572 & .0302096 & 31.8326 & $\mathbf{. 3 3 6 1 7 9}$ \\
\hline Bayeshrink & 33.1052 & .0304347 & 33.6789 & .690434 \\
\hline Neigh shrink & 33.1607 & .0296643 & $\mathbf{2 7 . 6 7 1 2}$ & 1.80169 \\
\hline Vishu shrink & 31.1211 & .0301562 & 31.4059 & 61.8985 \\
\hline Hybrid & $\mathbf{3 3 . 3 6 8 1}$ & .0321326 & 50.2313 & 2.14462 \\
\hline
\end{tabular}

B. Images corrupted by Speckle noise:

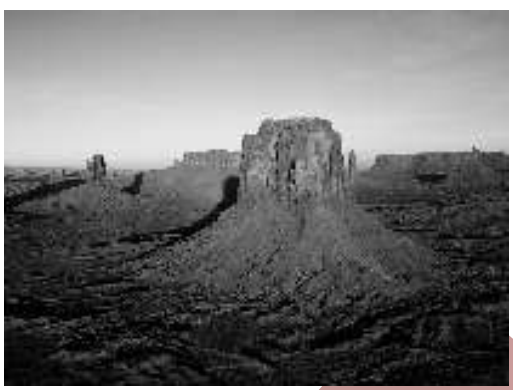

(a)

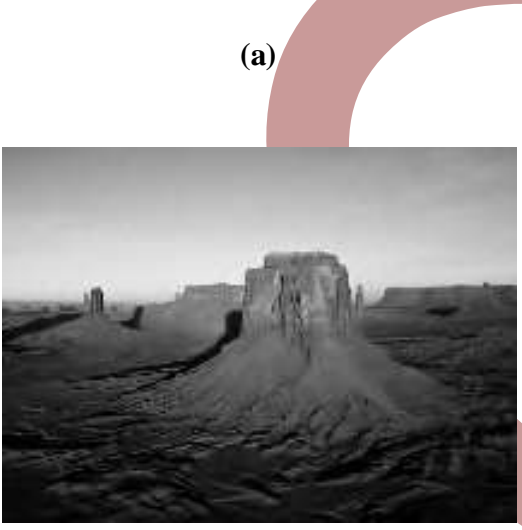

(d)

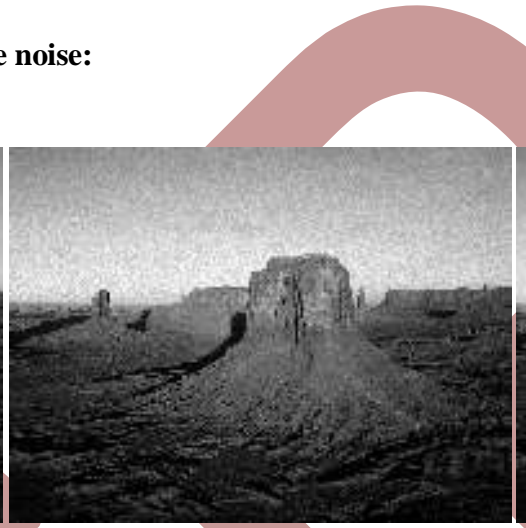

(b)

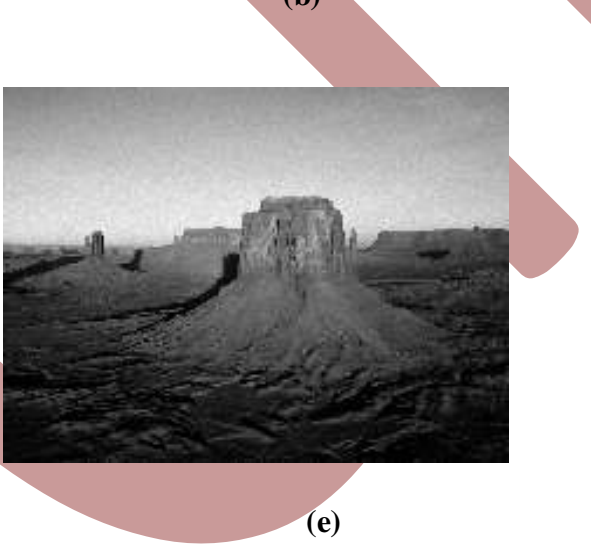

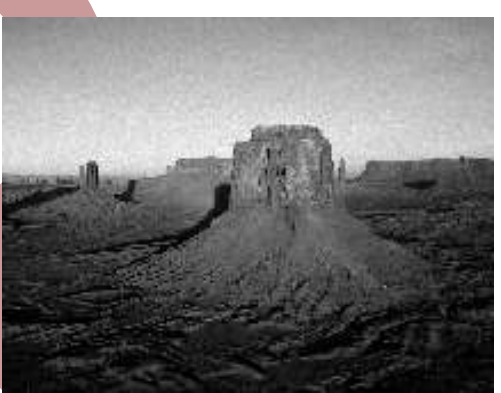

(c) (a) Original Image (b) Noisy Image,

(e) Hybrid method
Reconstructed images after applying (c) Bayeshrink

(d) Neighshrink

Table showing the performance of different filters

\begin{tabular}{|l|l|l|l|l|}
\hline Wavelets & PSNR & BER & MSE & TIME \\
\hline Median filter & 35.0082 & .0285648 & 26.5241 & $\mathbf{. 2 9 3 9 0 2}$ \\
\hline Wiener filter & 33.9989 & .0294128 & 25.8938 & .672223 \\
\hline Bayeshrink & 34.4354 & .0287127 & $\mathbf{1 1 . 7 3 6 7}$ & .4595 \\
\hline Neigh shrink & 33.8471 & .0295446 & 26.8145 & 61.1715 \\
\hline Vishu shrink & 35.7517 & .0294707 & 17.2945 & 1.52327 \\
\hline Hybrid & $\mathbf{3 5 . 1 3 0 7}$ & $\mathbf{. 0 2 8 4 6 5 1}$ & 19.953 & 23.3036 \\
\hline
\end{tabular}




\section{CONCLUSION}

In this paper, we proposed hybrid denoising technique for speckle \& additive white gausssian noise removal. This technique outperforms all other denoising algorithm taken like, Median, Bayeshrink, Neighshrink, Visushrink techniques and can deal with both low and high frequency components effectively. Hybrid technique is little slower as it take more time to process the image. After comparing the techniques, we observe that the Hybrid result is better than Bayeshrink techniques in terms of PSNR and BER. We recommend Bayeshrink in applications requiring faster processing times and Hybrid method where signal components are of more concern.

Futer work can be carried out by using better detailedsubband denoising by adopting neighborhood wavelet based thesholding.

\section{ACKNOWLEDGMENTS}

Our thanks to all the department of Electronics \& Communication, who helped us.

\section{REFERENCES}

[1] Gupta, S., Chauhan, R.C and Saxena, S.C., "Locally adaptive wavelet domain Bayesian Processor for denoising medical ultrasound images using speckle modeling based on Rayleigh distribution”, IEEE Proceedings.-Vision Image Signal Processing,Vol.152,No.1, February 2005.

[2] MIME Type Detection in Internet Explorer: Uploaded MIME Types (msdn.microsoft.com).

[3] JPEG File Layout and Format, "Designed and Managed by D Cube Software Technologies, Nagpur(India)" Last Revised: 5th July 2002.

[4] Gupta, S., Chauhan, R. and Saxena, S.(2004) "A wavelet based Statistical Approach for Speckle Reduction in Medical Ultrasound Images", Medical and Biomedical Engineering \& Computing, Vol. 42, No. 2, pp.189-192.

[5] Gupta, S., Kaur, L., Chauhan, R.C., and Saxena, S.C. "A versatile technique for visual enhancement of medical ultrasound images", Digital signals Processing, Vol 17 May 2007, PP 542-560.

[6] S. Grace Chang, Bin Yu and M. Vattereli, "Adaptive Wavelet Thresholding for Image Denoising and Compression", IEEE Trans. Image Processing, vol. 9, pp. 1532-1546, Sept. 2000.

[7] S. Grace Chang, Bin Yu and Martin Vetterli, “Adaptive Wavelet Thresholding for Image Denoising and Compression," IEEE Trans. Image Processing, Vol 9, No. 9, Sept 2000, pg 1532-1546.

[8] Kaur, L., Gupta,S., and Chauhan, R.C. (2000), "Image Denoising using Wavelet Thresholding", Deptt. of CSE,SLIET, Longowal.

[9] Aleksandra Pizurica,A., Wilfried Philips, W., Lemahieu, and Acheroy, M.,(2003), "A Versatile Wavelet Domain Noise Filtration Technique for Medical Imaging", IEEE Transactions on Medical imaging Vol.22, No.3, pp.323-331, March 2003.

[10] Achim, A., (2001), "Novel Bayesian Multiscale Method for Speckle Removal in Medical Ultrasound Images", IEEE
Transactions on Medical Imaging, Vol. 20, No. 8, August 2001.

[11] Sudha, S., Suresh, G.R. and Suknesh, R. "Speckle Noise Reduction in Ultrasound images using Context Based Adaptive Wavelet threshholding". IETE Journal of Research Vol 55 [issue3], 2009

[12] Zhang, M and Gunturk, B. (2008) "A New Image denoising Method based on the Bilateral Filter", ICASSP, IEEE, pp. 929-932[2].

[13] Gonzalez, Rafael C. and Woods, Richards E. (2006) "Digital Image Processing", Pearson Prentice Hall, New Delhi.

[14] D.L.Donoho (1995), "De-Noising by Soft-Thresholding", IEEE Transactions on Information theory, Vol.41, No.3, May1995.

[15] Gonzalez, R and Woods, R. (2006), "Digital Image Processing with MATLAB", Pearson Prentice Hall, New Delhi, pp. 417-425.

[16] Scott E Umbaugh, "Computer Vision and Image Processing”, Prentice Hall PTR, New Jersey, 1998.

[17] Acharya, Tinku. and Ray Ajoy K., "Image Processing Principles and Applications", 2005 edition A John Wiley \& Sons, Mc. Publication.

[18] Donoho, D.L. and Iain M. Johnstone, I.M. (1995) "Adapting to Unknown Smoothness via Wavelet Shrinkage," Journal of American statistical Association, 90(432):12001224, December 1995. 